\title{
Electrical Switching of Wetting States on Superhydrophobic Surfaces: A Route Towards Reversible Cassie-to-Wenzel Transitions
}

\author{
G. Manukyan, ${ }^{1,2}$ J. M. Oh, ${ }^{1}$ D. van den Ende, ${ }^{1}$ R. G. H. Lammertink, ${ }^{2}$ and F. Mugele ${ }^{1}$ \\ ${ }^{1}$ Physics of Complex Fluids, Fluidics and Interfaces, IMPACT and MESA+ Institute, Department of Science and Technology, \\ University of Twente, P.O. Box 217, 7500 AE Enschede, The Netherlands \\ ${ }^{2}$ Soft Matter, Fluidics and Interfaces, MESA+ Institute, Department of Science and Technology, University of Twente, \\ P.O. Box 217, 7500 AE Enschede, The Netherlands
}

(Received 17 March 2010; revised manuscript received 17 September 2010; published 7 January 2011)

We demonstrate that the equilibrium shape of the composite interface between superhydrophobic surfaces and drops in the superhydrophobic Cassie state under electrowetting is determined by the balance of the Maxwell stress and the Laplace pressure. Energy barriers due to pinning of contact lines at the edges of the hydrophobic pillars control the transition from the Cassie to the Wenzel state. Barriers due to the narrow gap between adjacent pillars control the lateral propagation of the Wenzel state. We demonstrate how reversible switching between the two wetting states can be achieved locally using suitable surface and electrode geometries.

DOI: 10.1103/PhysRevLett.106.014501

PACS numbers: 47.55.D-, 47.55.nb, 68.08.Bc

Superhydrophobic surfaces display remarkable properties including ultrahigh contact angles, ultralow contact angle hysteresis [1], hydrodynamic slip [2-4], and tunable optical diffraction $[5,6]$. These properties rely on the weak interaction between the liquid and the substrate due to the entrapment of air (or vapor) in the cavities of the rough surface topography. At the transition from the superhydrophobic "Cassie" state to the normal "Wenzel" wetting state, the cavities are filled with liquid resulting in a particularly intimate contact between solid and liquid. The resulting strong contrast in physical properties makes it desirable to design surfaces that allow for switching between the two wetting states. Various external control parameters have been used to trigger the transition from the Cassie to the Wenzel state, including hydrostatic pressure, optical and chemical stimuli (see [7] for a review), as well as electric fields [8-13]. In particular, the latter offers opportunities for fast and precise fine-tuning of the wetting state. Yet, a microscopic understanding of the mechanism controlling the properties of the superhydrophobic state under electrowetting (EW) conditions and, in particular, the stability limit of the Cassie state is lacking $[12,14]$. Such a detailed understanding will be crucial to reach the "holy grail" of reversible switching between these states, which so far has been limited to a few special cases $[10,13,15]$.

In this Letter we use interference microscopy to study the properties of liquid drops on superhydrophobic surfaces consisting of periodic arrays of micrometer-sized posts under EW. We identify the dimensionless parameters that control the reversible deflection of the interface at low voltage and we determine the physical processes controlling the stability of the Cassie state at higher voltage. Based on the resulting picture, we demonstrate local reversible switching between the Cassie and the Wenzel states.
The experimental setup consists of a millimeter-sized sessile drop (DI water) on a superhydrophobic surface made of a glass slide with a transparent indium tin oxide (ITO) electrode that is covered by a homogeneous layer of the equally transparent SU-8 photoresist (thickness $h_{d}=5 \mu \mathrm{m}$; dielectric constant $\varepsilon_{d}=3.2$, refractive index: $n=1.7)$ as shown in Fig. 1. On top of that homogeneous layer, square lattices of round pillars [diameter $2 a=$ $5 \mu \mathrm{m}$; height $h=(5 \pm 0,01) \mu \mathrm{m}]$ are fabricated from a second layer of SU-8 using photolithography with gap widths between the pillars of $d=3,5,8$, and $10 \mu \mathrm{m}$, respectively. The corresponding values of Wenzel's

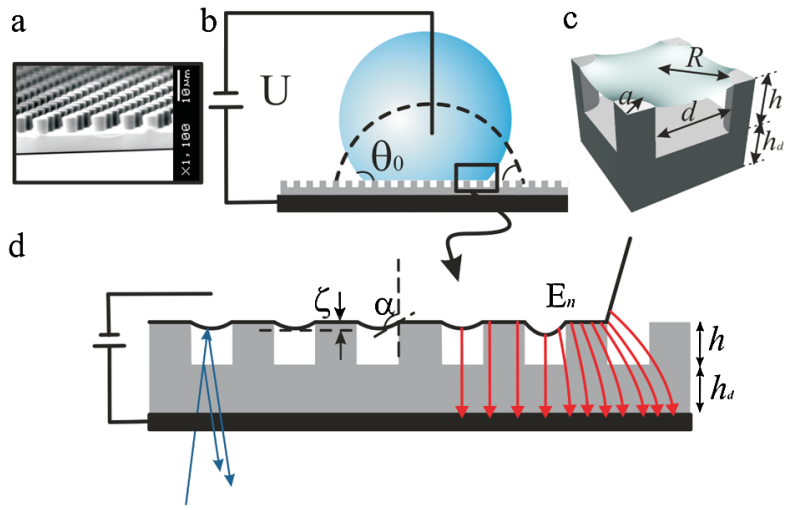

FIG. 1 (color online). System configurations. (a) Scanning electron microscope image of Teflon coated microstructure. (b) Electrowetting setup: a voltage $U$ is applied between a droplet and an electrode covered with a dielectric micropatterned surface. The contact area is monitored with an inverted microscope from the bottom through the superhydrophobic substrate. (c) The unit cell of microstructure. (d) Magnified sketch of the solid-liquid interaction area. Dark grey (blue) arrows: optical rays leading to interference. Light grey (red) arrows: electric field distribution (shown only close to contact line). 
TABLE I. Sample characteristics. $r$ : surface roughness, $f$ : fractional area, $\theta^{*}$ : critical angle for spontaneous propagation of the collapse, $\alpha_{c}$ : critical angle at the transition as obtained from the experiments, $U_{c}$ : critical voltage for single-pit filling, and $U^{*}$ : critical voltage for lateral propagation.

\begin{tabular}{ccccccc}
\hline \hline$d(\mu \mathrm{m})$ & $r$ & $f$ & $\theta^{*}$ & $\alpha_{c}$ & $U_{c}(V)$ & $U^{*}(V)(\beta=0,5)$ \\
\hline 3 & 2.23 & 0.307 & $103^{\circ}$ & $110^{\circ}$ & 225 & 270 \\
5 & 1.79 & 0.196 & $109^{\circ}$ & $115^{\circ}$ & 175 & 177 \\
8 & 1.46 & 0.116 & $116^{\circ}$ & $108^{\circ}$ & 123 & 91 \\
10 & 1.35 & 0.087 & $120^{\circ}$ & $109^{\circ}$ & 105 & 0 \\
\hline \hline
\end{tabular}

roughness factor $r=A_{\text {real }} / A$ ( $A_{\text {real }}$ : true solid surface area following the topography; $A$ : projected surface area) and of the fractional area $f$ of the pillars are given in Table I. The surfaces are hydrophobized by dip-coating from a $0.01 \%$ Teflon AF (Dupont) solution, which produces a contact angle of $\theta_{Y} \approx 115^{\circ}-120^{\circ}$ on a flat surface. Inserting the values of $\theta_{Y}, r$, and $f$ into the standard expressions [1] for the energies $G_{C}$ and $G_{W}$ per unit area of the Cassie and the Wenzel state, respectively, shows that $\left(G_{C}-G_{W}\right) / \sigma=$ $(r-f) \cos \theta_{Y}+(1-f)$ is positive, i.e., that the Wenzel state is thermodynamically favorable, for all pillar spacings but the smallest. The macroscopic contact angle is controlled by applying a voltage (here, dc) between the drop via an immersed metal wire and the ITO electrode. The drop is illuminated from below with monochromatic light $(\lambda=510 \mathrm{~nm})$ and observed in reflection mode using an inverted microscope (lens magnification: $4 \times$ and $20 \times$; numerical aperture: 0.13 and 0.40 ).

Upon linearly increasing the applied voltage the drops spread [see Fig. 2]. The SU-8 pillars appear dark, whereas the air-filled area in between is bright with lateral intensity variations due to interference of light that is partially reflected at the solid-air and at the liquid-air interface [see Fig. 1(d) and inset of Fig. 2]. As the voltage is increased and decreased, the interference pattern changes reversibly (see supplementary material [16] for movie M1) provided that the voltage is not too high. When the voltage is increased beyond a certain critical value $U_{c}$, some of the pits between pillars along the apparent contact line turn dark, indicating that these cavities are filled with water [see Fig. 2(e) and supplementary material [16] for movie M2]. The subsequent behavior depends strongly on the pillar spacing. For the smaller spacings ( 3 and $5 \mu \mathrm{m}$ ), individual cavities become water filled one by one [Figs. 2(f) and 2(g)]. The drop-substrate interface is then inhomogeneous, displaying a Wenzel-like state along the apparent contact line and a Cassie-like state elsewhere. For the larger pillar spacings water filling of pits spreads across the drop-substrate interface within a few milliseconds leading to a homogeneous Wenzel (see supplementary material [16] for movie M3).

To study the Cassie-to-Wenzel (CW) transition in more detail, we analyze the interference pattern originating from the deformable water-air interface. The deflection of the interface is maximal in the center of the pits and displays a saddle point between two adjacent pillars. To quantify the deformation of the interface, we extract the intensity averaged over the central area of 9 adjacent cavities as a function of the applied voltage (see supplementary material [16]) and extract the deflection $\zeta_{0}$ of the surface using plane wave interferometry with an accuracy better than $30 \mathrm{~nm}$. For $U<U_{c}$, the interface reversibly bends up and down many times indicating that the microscopic threephase contact lines along the edges of the pillars remain rigidly pinned. Upon increasing the pillar spacing, the

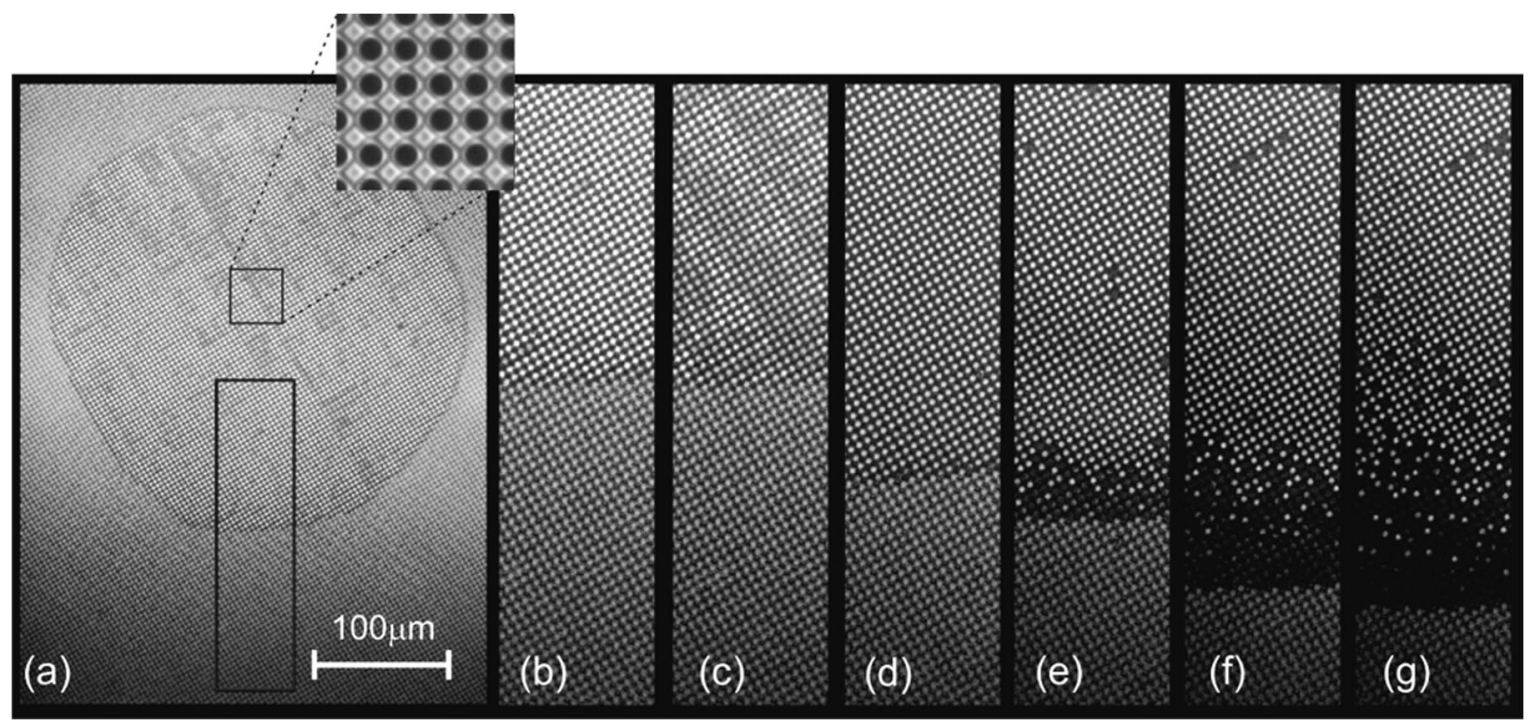

FIG. 2. Spreading of droplet on the superhydrophobic surface by ramping the voltage $(d=5 \mu \mathrm{m})$. Voltage increases from left to right: (a) $0 \mathrm{~V}$-the Cassie state, the entire contact area is shown: the droplet touches the tops of the microscopic pillars and rests on a cushion of air. (b)-(g) Magnified views of the black rectangle region in (a) at $U=0-250 \mathrm{~V}$ for every $50 \mathrm{~V}$, respectively. Black dots appeared near the contact line in $(\mathrm{d})-(\mathrm{g})$, representing the area in the Wenzel state. Inset: magnified view of the solid-liquid contact area at maximal deflection of menisci. 
deflection at any fixed voltage increases. Moreover, the critical voltage $U_{c}$ decreases (see Table I).

The deflection of the interface is due to the electric field $\vec{E}$ and the resulting Maxwell stress $\pi_{\mathrm{el}}=\varepsilon \varepsilon_{0} E^{2} / 2\left(\varepsilon_{0}\right.$ : vacuum permittivity; $\varepsilon$ : relative permittivity of medium in cavities in Cassie state, i.e., $\varepsilon=1$ for air) pulling outwards along the surface normal. In mechanical equilibrium, $\pi_{\mathrm{el}}$ is locally balanced by the Laplace pressure $\Delta p_{L}=\sigma \kappa$ of the curved interface, where $\sigma$ is the surface tension and $\kappa$ the mean curvature of the water-air interface

$$
\pi_{\mathrm{el}}=\Delta p_{L}(x, y)=\sigma \kappa(x, y) .
$$

Denoting the small local deflection of the water-air interface away from the flat configuration as $\zeta(x, y)$, we can write $\kappa=\partial^{2} \zeta / \partial x^{2}+\partial^{2} \zeta / \partial y^{2}$. Finding the equilibrium shape requires, in principle, a self-consistent calculation of both field distribution and surface profile [17]. For the present small defelctions, we simplify the electrical problem assuming a locally flat configuration leading to an electric field $E(x, y)=U / z_{\text {eff }}$ at the liquid surface. $z_{\text {eff }}=$ $H-\zeta(x, y)$ with $H=h+h_{d} \varepsilon / \varepsilon_{d} \approx 6.5 \mu \mathrm{m}$ is the effective dielectric spacing between the water-air interface and the electrode. Using the radius $R=\sqrt{2}(d / 2+a)-a$ [see Fig. 1(c)] as a characteristic scale in the $x, y$ direction and $H$ in the $z$ direction, Eq. (1) then assumes the dimensionless form

$$
\partial^{2} w / \partial u^{2}+\partial^{2} w / \partial v^{2}=\Lambda^{2} / w^{2},
$$

with $u=x / R, v=y / R$, and $w=z / H$. Here, $\Lambda=$ $R / H \eta^{1 / 2}\left(\eta=\varepsilon \varepsilon_{0} U^{2} / 2 \sigma H\right)$ is a dimensionless number measuring the relative strength of electrostatic and surface tension forces. Note that $\Lambda$ is given by the square root of the usual EW number $\eta$ (see e.g., [17]) modified by the ratio of the lateral over the vertical length scale. According to this analysis, the normalized deflection $\zeta_{0} / H$ should collapse if plotted versus the normalized parameter $\Lambda$, which is indeed the case [Fig. 3].

Equation (2) can be solved numerically using periodic boundary conditions in a quadrant of the unit cell with $u$, $v \in[0,(a+d / 2) / R]$ with pinning of the contact line along the edge of the pillar and boundary conditions implying pinning along the edge of the pillar $(w=1)$ and vanishing slopes $\partial w / \partial u=0$ for $u=0$ and $u=(a+$ $d / 2) / R$, and $\partial w / \partial v=0$ for $v=0$ and $v=(a+d / 2) / R$. The numerical solution reproduces the characteristic shape (see supplementary material [16]). For small $\Lambda$, the resulting deflection $\zeta_{0} / H$ agrees very well with the experimental results [see Fig. 3]. For larger $\Lambda$, the approximations in the model lead to a systematic underestimation of the deflection.

Interestingly, $\zeta_{0}$ can assume much larger values in the numerical calculations than in the experiments. The origin of this discrepancy is related to the pinning condition imposed at the edge of the pillars. Analyzing the numerical profiles for the largest $\Lambda$ reveals that the angle between the air-water interface and the vertical side wall of the pillars is

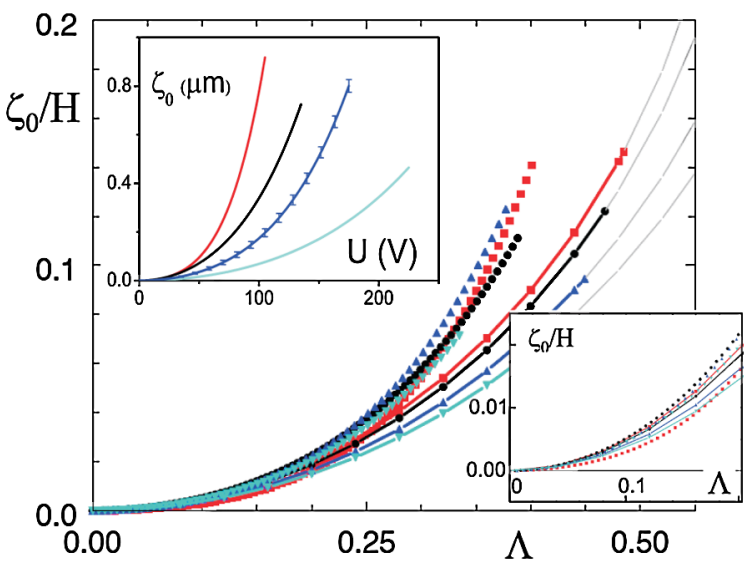

FIG. 3 (color online). Normalized deflection versus normalized electrostatic force (see text for details). Symbols: experimental data for variable post spacing $d=10 \mu \mathrm{m}$ [(red) squares], 8 (black circles), 5 [(blue) up triangles], and $3 \mu \mathrm{m}$ [(green) down triangles]. Symbols with lines: numerical calculations. The last symbols on the lines in model calculations, around $\Lambda \approx 0,4$, correspond to $\alpha_{c}=110^{\circ}$. Light grey dashed lines: numerically stable continuation of the solution. Inset (left): same experimental data in dimensional form $\zeta_{0}$ versus $U$. Error bars represent the typical standard deviation over $3 \times 3$ unit cells. Inset (right): Zoomed view of main panel.

$\alpha \approx 150^{\circ}$ which is much larger than the advancing angle $\theta_{\text {adv }}$ on the sidewall of the pillars. Hence, these solutions are not observable experimentally. The numerical profiles corresponding to the maximum deflection found in the experiments, on the other hand, display angles of $\alpha \approx$ $110^{\circ} \approx \theta_{\text {adv }}$. Hence, we conclude that the stability limit of the Cassie state is determined by contact line depinning, like for the pressure-driven $\mathrm{CW}$-transition $[6,18]$ (for sufficiently large aspect ratio of the pillars). This result is not trivial. For larger and slightly different geometry, Taylor cone-like instabilities can dominate over depinning [19].

To understand the preferential nucleation of the transition along the apparent contact line, we note that the electric field between the drop and the substrate is not homogeneous. For EW on flat substrates, it is well known that sharp edge effects give rise to the diverging electric fields in the vicinity of the contact line [16]. Numerical estimates for superhydrophobic surfaces qualitatively show the same effect. This explains the observed nucleation along the apparent contact line.

Why does the Wenzel state spread across the entire dropsubstrate interface for large pillar spacings but not for small ones? In order to propagate from one filled pit to the next, the liquid has to pass the gap between two adjacent pillars. It can only cross this energy barrier if the gain in electrostatic energy outweighs the additional cost in surface energy in the narrowest part of the gap. The variation of the energy upon advancing by a distance $\delta x$ within this gap is (width: $d$ )

$$
\delta G=\delta x\left\{(2 h+d)\left(\sigma_{\mathrm{sv}}-\sigma_{\mathrm{sl}}\right)+d\left[\sigma-\left(C_{C}-C_{W}\right) U^{2} / 2\right]\right\},
$$


a

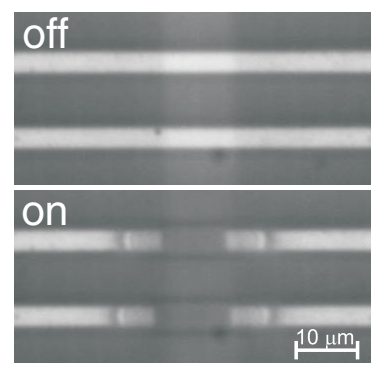

C

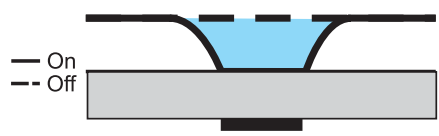

b

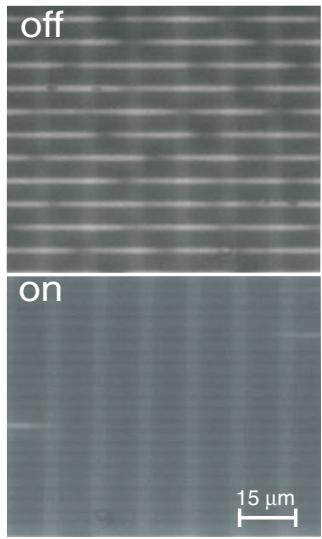

FIG. 4 (color online). (a) Snapshots of the liquid interface for single-stripe electrode (vertical) under two grooves (horizontal) at zero (top) and at maximum (bottom) voltage. (b) Surface with an array of parallel grooves (horizontal) and electrodes (vertical) with and without applied voltage. (c) Sketch of liquid vapor interface along a groove. Dashed line: zero voltage; solid: maximum voltage.

where $C_{C}$ and $C_{W}$ are the local capacitances in the Cassie and in the Wenzel state, respectively. Propagation occurs if $\delta G>0$. Writing the (positive) differential capacitance as $C_{W}-C_{C}=\beta \varepsilon_{0}\left(\varepsilon_{d} / h_{d}-\varepsilon / H\right)=\beta \varepsilon_{d} \varepsilon_{0} h / H h_{d}$, where $\beta$ is a correction factor of order unity accounting for the exact field distribution, this criterion yields a geometrydependent critical voltage $U^{*}$ above which propagation of the Wenzel state is energetically favorable

$$
U^{*}=U_{0} \sqrt{\frac{\varepsilon h_{d}}{\beta \varepsilon_{d} h}\left(\frac{\cos \theta_{Y}}{\cos \theta^{*}}-1\right)} .
$$

Here $\cos \theta^{*}=-d /(d+2 h)=-1+2 h /(2 h+d)$ represents the critical angle for spontaneous propagation at zero voltage [19] and $U_{0}=\left(2 \sigma H / \varepsilon \varepsilon_{0}\right)^{1 / 2} \approx 320 \mathrm{~V}$ is a characteristic voltage. Propagation of the Wenzel state is expected if $U_{c}>U^{*}$, whereas for $U_{c}<U^{*}$ collapsed areas of the drop-substrate interface and noncollapsed ones can coexist. $U_{c}$ is thus a measure of the energy barriers for the filling of individual pits, $U^{*}$ the one for lateral propagation. For values of $0.25<\beta<0.5$, propagation is predicted for the two largest pillar spacings but not for the two smallest ones, in agreement with the experiments.

Finally, we address the reversibility of the CW transition. Since applying a voltage always favors the Wenzel state, reversibility requires that the Wenzel state is unstable at zero voltage. This can be achieved by transforming the surface only locally into the Wenzel state above activated patterned electrodes, while other regions of the dropsubstrate interface remain vapor filled, such that the reverse switch only requires the motion of contact lines but not the nucleation of vapor "from scratch."
The success of this concept is demonstrated using a surface with parallel rectangular grooves in the horizontal direction and a perpendicular stripe electrode [see Fig. 4(a)]. Upon activating the electrode, the liquid surface is pulled down only at the position of the electrode. Upon turning off the voltage, the liquid switches back to the Cassie state (see also supplementary material [16] for movie M4). Combining several parallel stripe electrodes, it is possible to reversibly switch large parts of the liquidsubstrate interface from the Cassie-like to the Wenzel-like state and back [Fig. 4(b)].

This concept opens up avenues towards a broader class of two- and three-dimensional functionalized structures for detailed control, actuation, and release of fluids.

We thank Matthias Wessling for fruitful discussions. This work was supported by the joint Priority Program Micro- and Nanofluidics of the research institutes MESA+ and Impact at Twente University and by NanoNed, the Dutch initiative for Nanotechnology (TSF 7134).

[1] D. Quere, Rep. Prog. Phys. 68, 2495 (2005).

[2] C. Choi and C.J. Kim, Phys. Rev. Lett. 96, 066001 (2006).

[3] P. Joseph et al., Phys. Rev. Lett. 97, 156104 (2006).

[4] A. Steinberger, C. Cottin-Bizonne, P. Kleimann, and E. Charlaix, Nature Mater. 6, 665 (2007).

[5] H. Rathgen et al., Phys. Rev. Lett. 99214501 (2007).

[6] H. Rathgen and F. Mugele, Faraday Discuss. 146, 49 (2010).

[7] N. Verplanck, Y. Coffinier, V. Thomy, and R. Boukherroub, Nano. Res. Lett. 2, 577 (2007).

[8] D. L. Herbertson et al., Sens. Actuators. A Phys. 130-131, 189 (2006).

[9] T. N. Krupenkin, J. A. Taylor, T. M. Schneider, and S. Yang, Langmuir 20, 3824 (2004).

[10] M. S. Dhindsa et al., Langmuir 22, 9030 (2006).

[11] N. Verplanck et al., Nano Lett. 7, 813 (2007).

[12] A. Ahuja et al., Langmuir 24, 9(2008).

[13] T. N. Krupenkin et al., Langmuir 23, 9128 (2007).

[14] V. Bahadur and S. V. Garimella, Langmuir 23, 4918 (2007).

[15] J. B. Boreyko and C. Chen, Phys. Rev. Lett. 103, 174502 (2009).

[16] See supplementary material at http://link.aps.org/ supplemental/10.1103/PhysRevLett.106.014501 for movies.

[17] J. Buehrle, S. Herminghaus, and F. Mugele, Phys. Rev. Lett. 91, 086101 (2003).

[18] F. Mugele and J.-C. Baret, J. Phys. Condens. Matter 17, R705 (2005).

[19] J. M. Oh et al. (to be published).

[20] M. Sbragaglia et al., Phys. Rev. Lett. 99, 156001 (2007). 